DOI: $10.15593 / 2224-9982 / 2018.52 .05$

УДК 629.735.33

\author{
А.Е. Кишалов, Н.А. Ключев
}

Уфимский государственный авиационный технический университет, Уфа, Россия

\author{
МЕТОДИКА ЗD ЧИСЛЕННОГО ТЕРМОГАЗОДИНАМИЧЕСКОГО \\ МОДЕЛИРОВАНИЯ АЭРОДИНАМИЧЕСКИХ ХАРАКТЕРИСТИК \\ ВЫСОКОМАНЕВРЕННЫХ САМОЛЕТОВ У ПОКОЛЕНИЯ
}

Рассматривается методика 3D численного термогазодинамического моделирования аэродинамических характеристик планеров современных военных высокоманевренных перспективных самолетов V поколения отечественного и зарубежного производства (на примере самолетов T-50 и F-35B), анализируется их лобовое сопротивление на различных высотах и скоростях полета. Исследование включает в себя три этапа. На первом этапе были построены 3D твердотельные модели самолетов при помощи CAD-системы Autodesk Inventor 2016, которые в дальнейшем были импортированы в программный комплекс ANSYS 17.0 CFX, в котором произведено моделирование процесса обтекания планеров самолетов потоком воздуха на различных высотах и скоростях полета (для угла атаки $0^{\circ}$ ). В результате моделирования получены значения лобового сопротивления планеров, а также исследованы зависимости изменения относительного сопротивления $R / P_{\max }$ от высоты и скорости полета. На данном этапе моделирование проводилось без учета работы двигателей, что привело к несколько завышенным значениям лобового сопротивления. На втором этапе для учета реактивной тяги двигателей (на различных высотах и скоростях) и уточнения результатов моделирования рассчитаны высотно-скоростные характеристики этих двигателей в CAE-системе DVIGw. Произведена идентификация моделей двигателей на максимальном и полном форсированном взлетных режимах. Результаты моделирования двигателей использованы в качестве граничных условий для третьего этапа, в котором на основе рассчитанных высотноскоростных характеристик в ANSYS CFX моделировались течение потока, входящего в воздухозаборник (входной импульс), и реактивная струя, выходящая из сопла (выходной импульс). В результате серии уточняющих расчетов получены новые значения лобового сопротивления самолетов. Проанализировано изменение результатов моделирования лобового сопротивления самолетов с учетом реактивной тяги двигателей по сравнению с моделированием на первом этапе.

Ключевые слова: лобовое сопротивление, Inventor, термогазодинамическое моделирование, ANSYS CFX, реактивная тяга, идентификация, имитационное моделирование, DVIGw, коэффициент сопротивления, высотно-скоростные характеристики.

\author{
A.E. Kishalov, N.A. Klyuchev
}

Ufa State Aviation Technical University (USATU), Ufa, Russian Federation

\title{
METHODS OF 3D NUMERICAL THERMOGASDYNAMIC MODELS OF AERODYNAMIC CHARACTERISTICS OF HIGHLY MANOEUVRABILITY V GENERATION AIRCRAFT
}

\begin{abstract}
The technique of 3D numerical thermogasdynamic modeling of the aerodynamic characteristics of gliders of modern military high manoeuvrable perspective V-generation aircrafts of domestic and foreign production (on the example of T-50 and F-35B aircrafts) and analysis of their aerodynamic drag at different altitudes and flight speeds are considered. The research includes three stages. At the first stage, 3D solid models of aircraft were built using the Autodesk Inventor 2016 CAD system, which were later imported into the ANSYS 17.0 CFX software package, which simulated the air flow around airplanes at various heights and flight speeds (for the angle of attack $0^{\circ}$ ). As a result of the simulation, the values of the aerodynamic drag of the gliders were obtained, and the dependence of the change in the relative aerodynamic drag $R / P_{\max }$ on the altitude and flight speed was investigated. At this stage, the simulation was carried out without taking into account the work of the engines, which led to some overestimated values of the aerodynamic drag. At the second stage, in order to take into account, the reactive thrust of the engines (at different altitudes and speeds) and refine the simulation results, the altitude-speed characteristics of these engines in the CAE system of DVIGw were calculated. The identification of engine models at the maximum and full forced take-off modes was made. The results of engine modeling were used as the boundary conditions for the third stage, in which the flow of air intake (input impulse) and the jet coming from the nozzle (output pulse) were modeled on the basis of the calculated altitude-speed characteristics in the ANSYS CFX. As a result of a series of refinement calculations, new values of aerodynamic
\end{abstract}


drag of aircraft were obtained. The change in the results of simulation of the frontal resistance of aircraft with the reactive thrust of the engines compared with the simulation in the first stage is analyzed.

Keywords: head resistance, Inventor, thermogasdynamic modeling, ANSYS CFX, jet thrust, identification, imitating modeling, DVIGw, resistance coefficient, high-speed characteristics.

\section{Введение}

Начиная с 1980-х гг. и по настоящее время во всем мире активно ведутся работы по созданию самолетов V поколения. Новое поколение самолетов отличается от предыдущего существенным увеличением удельной тяги, уменьшением расхода топлива, кардинальным уменьшением радиолокационной и инфракрасной заметности самолета, высокой боевой эффективностью, а также полетом на сверхзвуковых скоростях без использования форсажа. На сегодняшний день к военным самолетам V поколения можно отнести малозаметный многоцелевой истребитель F-22 Raptor и малозаметный истребитель-бомбардировщик F-35 (в модификациях $A, B$ и $C$ ), разработанные компанией Lockheed Martin CША, а также перспективный авиационный комплекс фронтовой авиации (ПАК ФА) Т-50 (Су-57) разработанный «ОКБ Сухого» РФ $[1,2]$.

Одним из важнейших показателей совершенства летательного аппарата (ЛА) является его лобовое сопротивление. Для того чтобы увеличить скорость ЛА, необходимо либо уменьшить его лобовое сопротивление путем совершенствования планера, либо увеличить тягу силовой установки, в качестве которой для самолетов $\mathrm{V}$ поколения используется турбореактивный двухконтурный двигатель с форсажной камерой сгорания и со смешением потоков (ТРДДФсм). Данное исследование посвящено моделированию обтекания планеров самолетов V поколения и определению их лобового сопротивления при помощи 3D численного термогазодинамического моделирования в ANSYS CFX [2-4].

\section{Моделирование лобового сопротивления планеров ЛА}

На первом этапе исследования проведено 3D численное термогазодинамическое моделирование лобового сопротивления самолетов T-50 и F-35 на различных высотах и скоростях полета в программном продукте ANSYS 17.0 CFX. Данное исследование проведено без учета входного и выходного импульса двигателей, что приводит к некоторой погрешности моделирования обтекания планера и определения лобового сопротивления самолетов [3].

Были построены 3D-модели самолетов Т-50 и F-35B в CAD системе Autodesk Inventor 2016 (рис. 1). При построении были допущены некоторые одинаковые для обеих моделей упрощения в области воздухозаборника, профиля крыла и сопла [6].

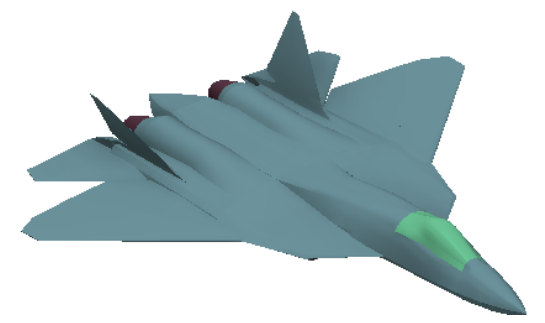

$a$

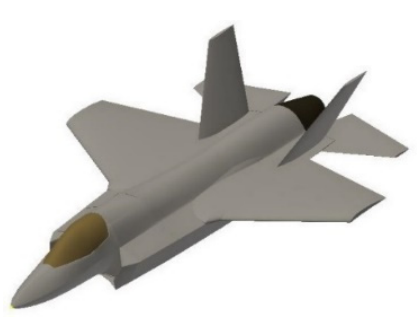

$\sigma$

Рис. 1. 3D-модели самолетов, построенные в Autodesk Inventor: $a$ - T-50; $\sigma$ - F-35B

B ANSYS Mesh на геометрических моделях построена конечно-элементная тетраэдрическая неструктурированная сетка с количеством элементов порядка 3 млн, с размером одного элемента порядка 700 мм. Для повышения точности расчетов сетка замельчена в области самолета с размерами элемента 60 мм для T-50 и 25 мм для F-35. Для лучшего решения в области 
пограничного слоя на твердых стенках модели построены структурированные призматические слои в количестве 15 штук и общей толщиной 70 мм (рис. 2). Для уменьшения времени расчета использовались только половины моделей [3, 7].

Далее в ANSYS CFX заданы граничные условия (рис. 3). Моделирование произведено с числами Маха полета от 0,2 до 1 для высот 0,2, 10 и 18 км над уровнем моря (18 км - практический потолок для F-35B) [8].

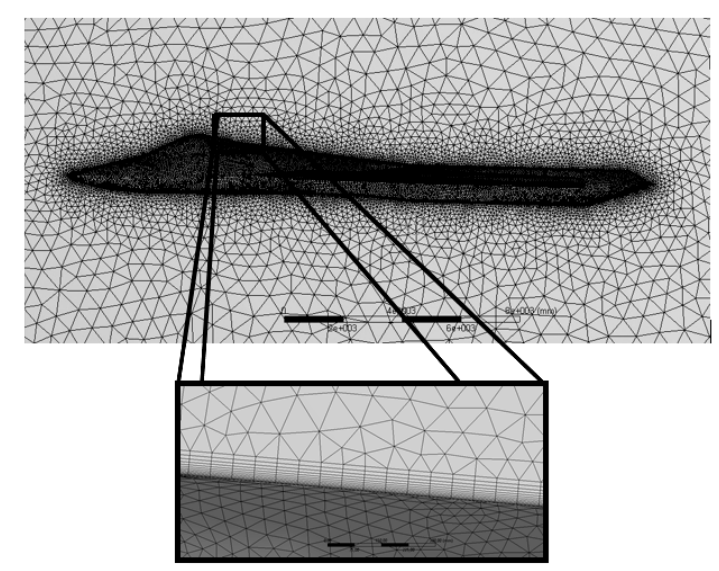

Рис. 2. Конечно-элементная сетка модели самолета Т-50

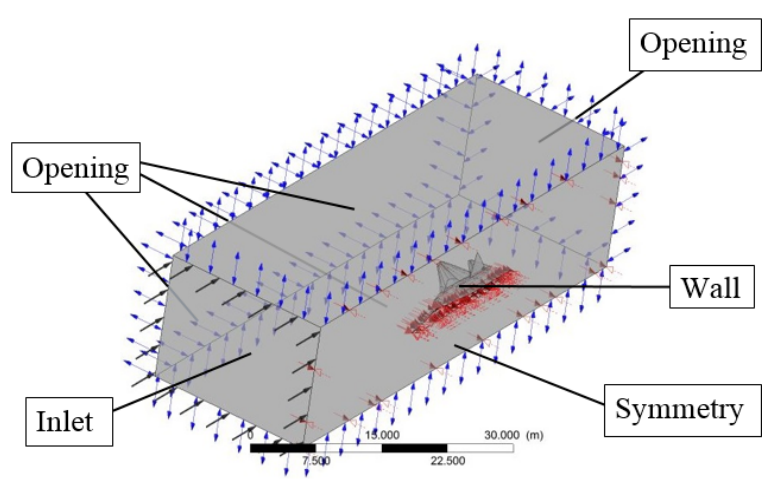

Рис. 3. Граничные условия в CFX на примере самолета Т-50

Для задания граничных условий на входе в модель определены параметры атмосферы на выбранных высотах ${ }^{1}$, вычислены значения скорости звука и скорости потока (табл. 1) [3].

В ходе исследования проведено 28 расчетов (по 14 на каждую модель самолета). В процессе расчета сходимость решения основных уравнений составила $10^{-4}$. Дисбаланс сходимости уравнений во всех расчетах порядка 0,1\% [8].

Таблица 1

Праметры граничных условий

\begin{tabular}{|c|c|c|c|c|c|}
\hline$H$, км & $p_{\mathrm{H}}, \Pi \mathrm{a}$ & $T_{\mathrm{H}}, \mathrm{K}$ & $\mathrm{M}$ & $a, \mathrm{M} / \mathrm{c}$ & $W, \mathrm{~m} / \mathrm{c}$ \\
\hline \multirow{4}{*}{0,2} & \multirow{4}{*}{98945} & \multirow{4}{*}{286,8} & 0,2 & \multirow{4}{*}{339,5} & 67,90 \\
\hline & & & 0,4 & & 135,8 \\
\hline & & & 0,6 & & 203,7 \\
\hline & & & 0,8 & & 271,6 \\
\hline \multirow{5}{*}{10} & \multirow{5}{*}{26500} & \multirow{5}{*}{223,2} & 0,2 & \multirow{5}{*}{229,5} & 59,90 \\
\hline & & & 0,4 & & 119,8 \\
\hline & & & 0,6 & & 179,7 \\
\hline & & & 0,8 & & 239,6 \\
\hline & & & 1,0 & & 299,5 \\
\hline \multirow{5}{*}{18} & \multirow{5}{*}{7565} & \multirow{5}{*}{216,6} & 0,2 & \multirow{5}{*}{295,0} & 59,01 \\
\hline & & & 0,4 & & 118,0 \\
\hline & & & $\overline{0,6}$ & & 177,0 \\
\hline & & & 0,8 & & 236,0 \\
\hline & & & 1,0 & & 295,0 \\
\hline
\end{tabular}

\footnotetext{
${ }^{1}$ ГОСТ 4401-81. Атмосфера стандартная. Параметры. М., 1981. 181 с.
} 
Для визуализации обтекания моделей потоком воздуха на рис. 4 и 5 приведены линии тока и векторы скоростей для $\mathrm{M}=0,6$ и на высоте $H=10$ км.

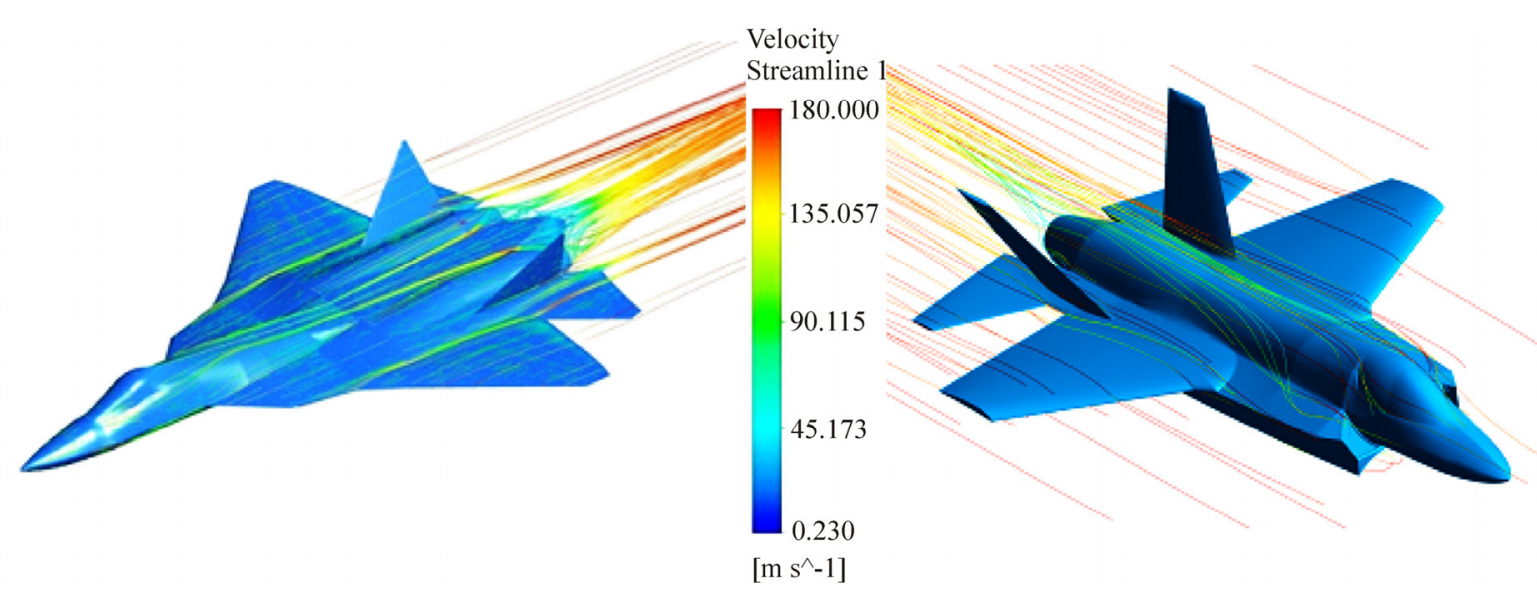

$a$

6

Рис. 4. Линии тока на моделях самолетов: $a-\mathrm{T}-50 ; \sigma-\mathrm{F}-35 \mathrm{~B}$

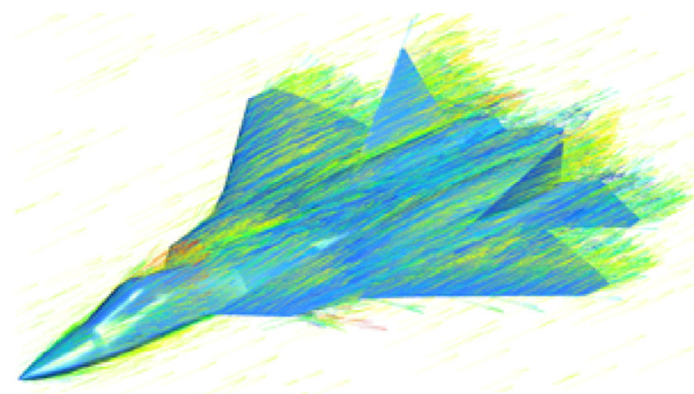

$a$

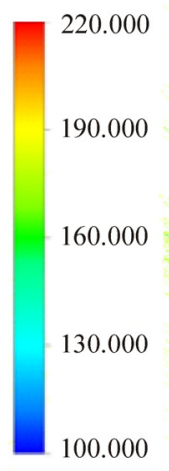

$\left[\mathrm{m} \mathrm{s}^{\wedge}-1\right]$

Рис. 5. Векторы скоростей на моделях самолетов: $a-\mathrm{T}-50 ; 6-$ F-35В

На рис. 6 приведены объемные поля температур на моделях для $\mathrm{M}=1$ и на высоте $H=18$ км.

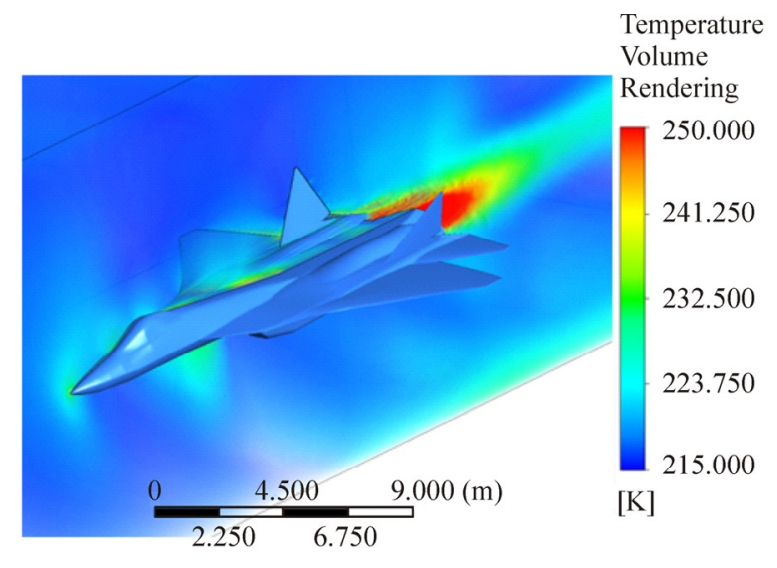

$a$

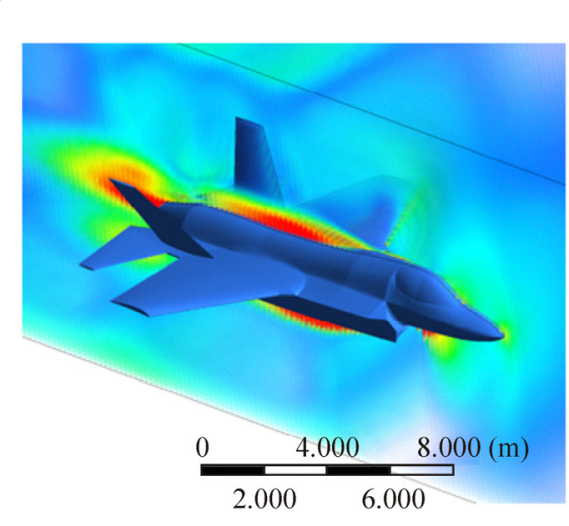

$\sigma$

Рис. 6. Объемные поля температур на моделях при $\mathrm{M}=1$ и $H=18$ км: $a-\mathrm{T}-50 ; \sigma-\mathrm{F}-35 \mathrm{~B}$ 
Для низких скоростей полета, на которых сжимаемостью воздуха можно пренебречь, коэффициент лобового сопротивления самолетов $\xi$ зависит только от геометрии модели и не зависит от параметров потока. Коэффициент сопротивления можно рассчитать как отношение изменения полного давления к динамическому напору

$$
\xi=\Delta p^{*} / \frac{\rho W^{2}}{2} .
$$

Для каждой модели определены коэффициенты сопротивления и построены зависимости $\xi$ от чисел Маха и высоты (рис. 7) [9].

На рисунке видно, как коэффициенты сопротивления на разных высотах для каждой из моделей самолетов практически совпадают ( $\xi$ не зависит от скорости и других параметров потока), но при приближении скорости полета к скорости звука начинает появляться волновое сопротивление ЛА и сопротивление моделей резко увеличивается.

На рис. 8 представлена зависимость относительного сопротивления моделей самолетов (сопротивление потока к тяге на взлетном максимальном режиме самолетов $R / P_{\max }$ ) от разных чисел Маха при различных высотах полета.

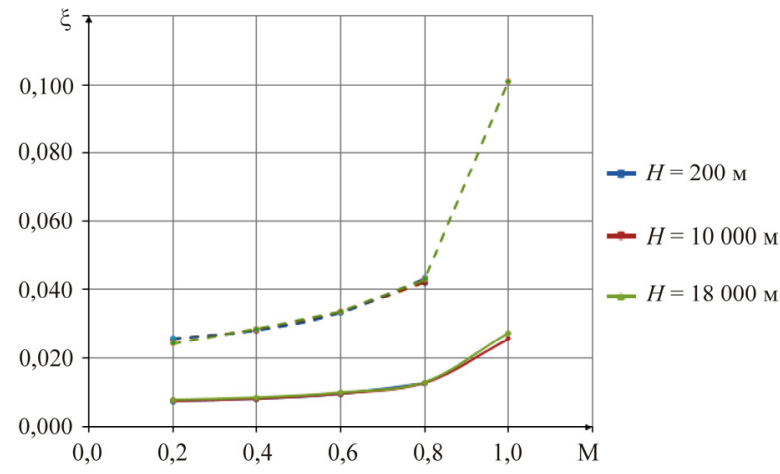

Рис. 7. Зависимость коэффициента сопротивления моделей $\xi$ от скорости М и высоты полета $H$ : - T-50-- - F-35

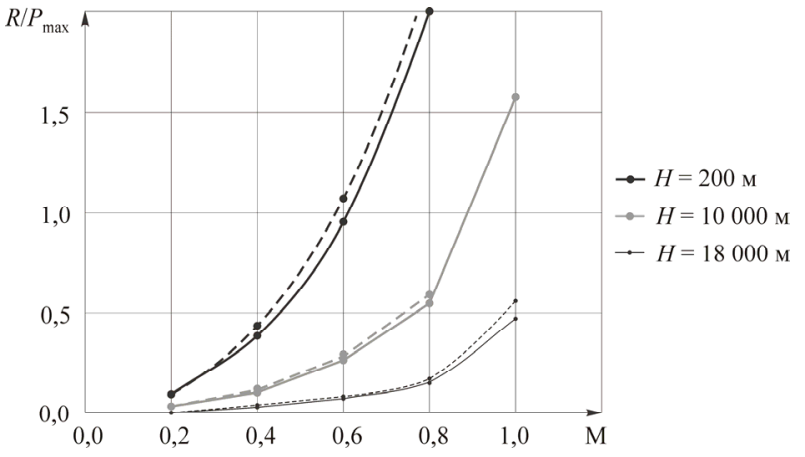

Рис. 8. Зависимость $R / P_{\max }$ от скорости М и высоты полета $H$ : — T-50 - - F-35

На рисунке видно, что с увеличением высоты полета относительное сопротивление падает, а с увеличением скорости возрастает. В области выше линии $R / P_{\max }=1$ (линия условная, так как в зависимости от высоты и скорости полета двигатели вырабатывают различную тягу) полет ЛА с данным планером без включения форсажа невозможен. Зависимости для самолетов T-50 и F-35B практически совпадают, что говорит о примерно равной тяговооруженности (оба самолета относятся к V поколению).

\section{Моделирование высотно-скоростных характеристик двигателей}

На следующем этапе рассчитывались высотно-скоростные характеристики двигателей $(\mathrm{BCX})$ для моделирования реактивной тяги этих двигателей. Для более точного моделирования обтекания планера самолета и определения его лобового сопротивления необходимо учитывать влияние струи воздуха, входящего в воздухозаборник двигателя, и реактивной струи, истекающей из сопла, уменьшающей данное сопротивление [10].

На сегодняшний день к ТРДДФсм для высокоманевренных военных самолетов V поколения относятся: F-119-PW-100 (истребитель F-22 Raptor) и F-135 (истребитель-бомбардировщик F-35 Lightning II) фирмы Pratt \& Whitney, АЛ-41Ф1 и АЛ-41Ф2 (Т-50 ПАК ФА), разработанные в НПО «Сатурн». Параметры данных ТРДДФсм на взлетном режиме $(H=0$ м, $\mathrm{M}=0$ ) и летные характеристики самолетов представлены в табл. 2 [2]. 
ТРДДФсм и характеристики самолетов

\begin{tabular}{|c|c|c|c|c|}
\hline \multirow{4}{*}{ Характеристики } & \multicolumn{4}{|c|}{ Самолет } \\
\hline & \multicolumn{2}{|c|}{$\mathrm{T}-50$} & F-22 & F-35 \\
\hline & \multicolumn{4}{|c|}{ Двигатель } \\
\hline & АЛ-41Ф1 & АЛ-41Ф2 & F-119 & F-135 \\
\hline Максимальная бесфорсажная скорость полета, $\mathrm{M}_{\max }$ & 1,20 & 1,20 & 1,22 & 0,80 \\
\hline Максимальная скорость полета на форсаже, $\mathrm{M}_{\Phi}$ & 2,10 & 2,50 & 2,25 & 1,60 \\
\hline Практический потолок, $H_{\max }, \mathrm{M}$ & 20000 & 21000 & 20000 & 18000 \\
\hline Тяга на форсаже $R_{\phi}$, кH & 140,000 & 160,000 & 155,600 & 191,200 \\
\hline Тяга на максимальном режиме $R_{\max }$, кH & 87,900 & 110,800 & 103,000 & 125,400 \\
\hline Удельный расход топлива на форсаже $C_{\text {уд. }}$, кг/Н·ч & 0,192 & 0,178 & 0,198 & 0,177 \\
\hline $\begin{array}{l}\text { Бесфорсажный удельный расход топлива, тяга на максималь- } \\
\text { ном режиме } C_{\text {уд } \max ,}, \text { кг/Н·ч }\end{array}$ & 0,075 & 0,067 & 0,089 & 0,087 \\
\hline Расход воздуха $G_{\mathrm{B}}$, кг/с & 120,6 & 124,2 & 125,0 & 159,0 \\
\hline Степень двухконтурности $m$ & 0,54 & 0,26 & 0,20 & 0,57 \\
\hline Степень повышения давления в КНД $\pi_{\text {Кнд }}$ & 3,88 & 5,21 & 4,50 & 3,70 \\
\hline Степень повышения давления в КВД $\pi_{\text {Квд }}$ & 6,62 & 5,37 & 7,78 & 7,75 \\
\hline Суммарная степень повышения давления $\pi_{\Sigma}$ & 25,67 & 28,00 & 35,00 & 28,00 \\
\hline Температура газа перед турбиной $T_{\text {г max }}, \mathrm{K}$ & 1765 & 1800 & 1866 & 1927 \\
\hline
\end{tabular}

Расчет ВСХ двигателей произведен в системе имитационного термогазодинамического моделирования (СИМ) DVIGw. Так как все рассматриваемые двигатели выполнены по схеме ТРДДФсм, то их топологические модели одинаковы (рис. 9) [11].

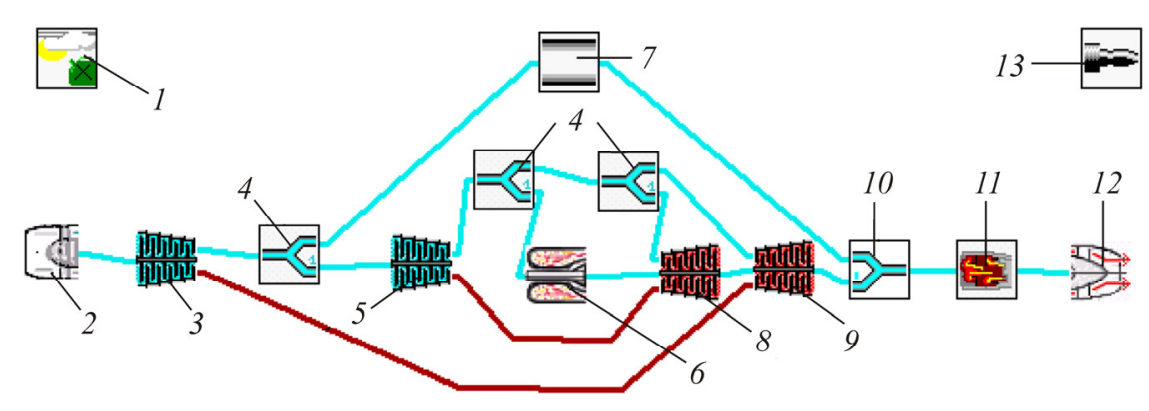

Рис. 9. Топологическая модель ТРДДФсм АЛ-41Ф1 в СИМ DVIGw: 1 - начальные условия; 2 - входное устройство; 3 - КНД; 4 - отбор воздуха; 5 - КВД; 6 - КС; 7 - канал; 8 - ТВД; 9 - ТНД; 10 - смеситель; 11 - форсажная камера; 12 - реактивное сопло; 13 - общие результаты

Для каждой модели двигателя по заданным значениям основных параметров рабочего процесса проводилась идентификация модели на взлетном форсированном и максимальном бесфорсажном режимах $(H=0, \mathrm{M}=0)$. Погрешность моделирования основных параметров двигателя по сравнению с данными из открытых источников составила не более $0,1 \%[12,13]$.

При моделировании ВСХ двигателей принят закон управления двигателем с поддержанием постоянной приведенной частоты вращения ротора высокого давления. При этом не учитывалось переключение системы автоматического управления (САУ) двигателем на другие законы регулирования, а также ограничения по высоте и скорости полета. Для всех двигателей ВСХ рассчитывались в диапазоне скоростей от 0 до $1,2 \mathrm{M}$ с шагом $0,2 \mathrm{M}$ и в диапазоне высот от 0 до 20000 м с шагом 2000 м. При моделировании использовалась стандартная обезразмеренная 
характеристика компрессора kom10 - это также является некоторым приближением, так как у каждого из рассматриваемых двигателей свои компрессоры низкого и высокого давлений со своими уникальными характеристиками [14-16].

На рис. 10 для рассматриваемых ТРДДФсм на максимальном бесфорсажном режиме приведено изменение тяги и удельного расхода топлива в зависимости от скорости полета с расслоением для трех высот ( $H=0, H=10000, H=20000$ м).
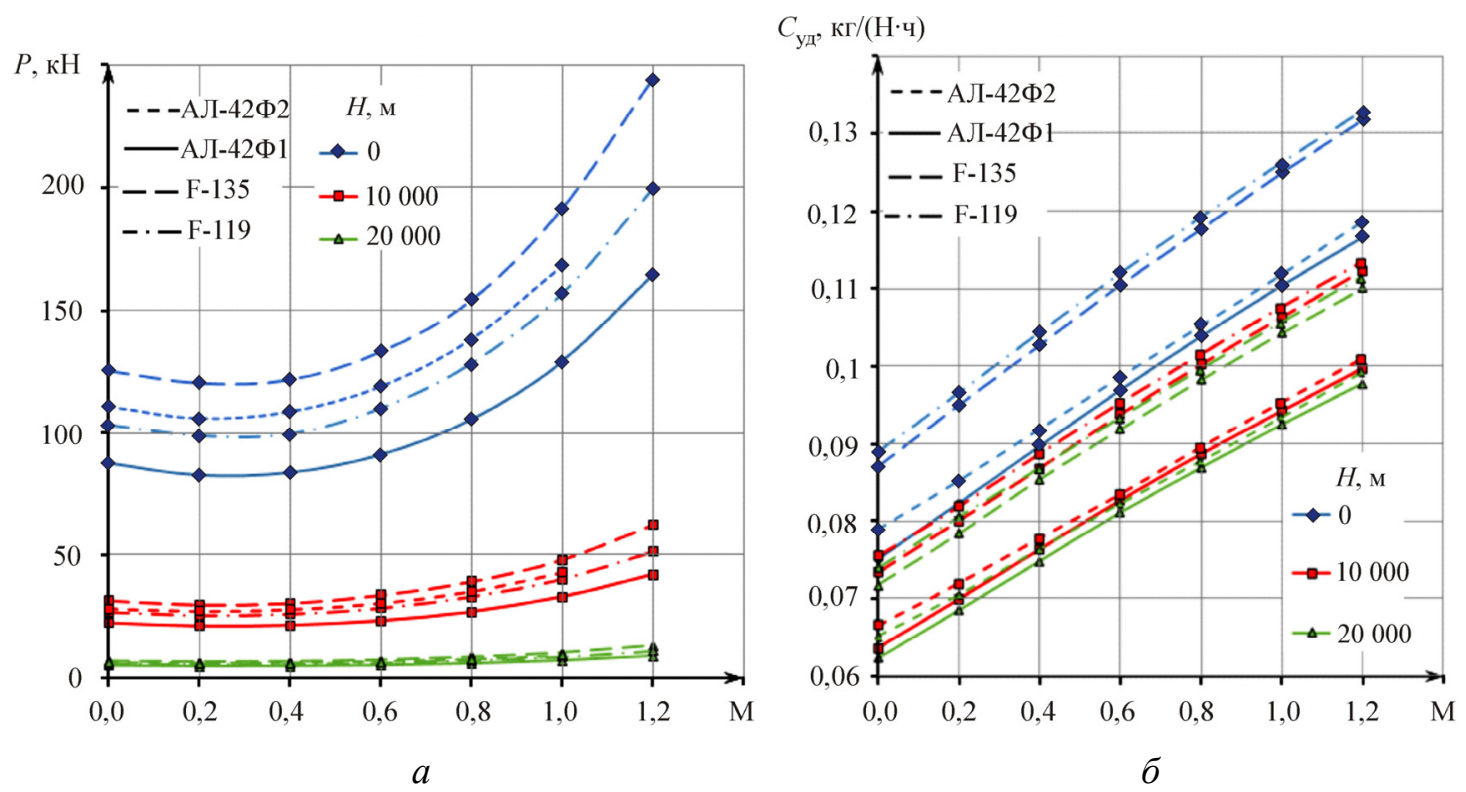

Рис. 10. Зависимость тяги от высоты и скорости полета $(a)$; зависимость удельного расхода топлива от высоты и скорости полета (б)

\section{Моделирование лобового сопротивления ЛА с учетом работы двигателей}

На заключительном этапе исследования для более точного расчета лобового сопротивления ЛА в ANSYS CFX, по данным расчета ВCX, установлены новые граничные условия (рис. 11), которые учитывают входящий поток воздуха в воздухозаборник и выходящую реактивную струю из сопла, что в целом позволяет моделировать работу двигателей.

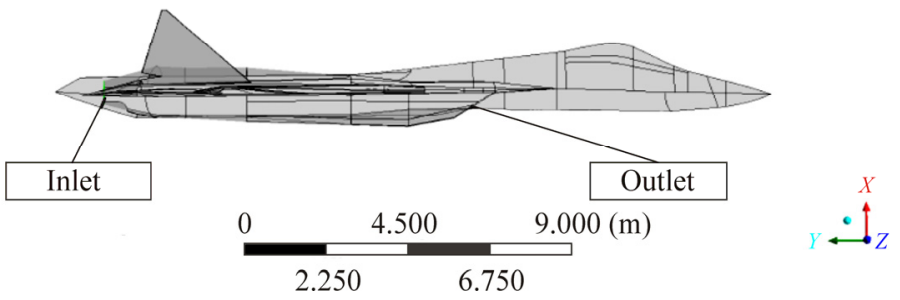

Рис. 11. Граничные условия, учитывающие работу двигателей

Для получения достоверной тяги двигателя при моделировании ВСХ определены входной импульс в воздухозаборнике (Outlet - выход из расчетной зоны) и выходной импульс из сопла модели (Inlet - вход в расчетную зону) для трех высот 200 м, 10 и 20 км при числах Маха от 0,2 до 1 с шагом 0,2 (табл. 3) [17, 18]. 
Таблица 3

Праметры граничных условий для Т-50 с ТРДДФсм АЛ-41Ф2

\begin{tabular}{|c|c|c|c|c|c|c|c|}
\hline$H$, км & $\mathrm{M}$ & $m, \mathrm{\kappa г} / \mathrm{c}$ & $W, \mathrm{~m} / \mathrm{c}$ & $P_{\max }, \mathrm{\kappa H}$ & $T_{\mathrm{c}}, \mathrm{K}$ & $W_{\text {вз }}, \mathrm{M} / \mathrm{c}$ & $W_{\mathrm{c}}, \mathrm{M} / \mathrm{c}$ \\
\hline \multirow{4}{*}{0,2} & 0,2 & 126,95 & 67,90 & 105,932 & 1022,57 & 95,932 & 787,482 \\
\cline { 2 - 8 } & 0,4 & 136,06 & 135,8 & 108,672 & 1050,49 & 140,397 & 831,988 \\
\cline { 2 - 8 } & 0,6 & 152,68 & 203,7 & 118,869 & 1092,26 & 182,164 & 906,598 \\
\cline { 2 - 8 } & 0,8 & 178,94 & 271,6 & 138,096 & 1147,63 & 273,133 & 1159,905 \\
\hline \multirow{4}{*}{10} & 0,2 & 37,77 & 59,90 & 26,978 & 766,08 & 84,677 & 674,611 \\
\cline { 2 - 8 } & 0,4 & 40,49 & 119,8 & 27,664 & 787,49 & 123,976 & 713,234 \\
\cline { 2 - 8 } & 0,6 & 45,50 & 179,7 & 30,172 & 816,69 & 160,980 & 776,168 \\
\cline { 2 - 8 } & 0,8 & 52,63 & 239,6 & 35,031 & 868,46 & 199,926 & 870,655 \\
\cline { 2 - 8 } & 1,0 & 63,50 & 299,5 & 42,884 & 928,28 & 245,494 & 998,655 \\
\hline \multirow{4}{*}{18} & 0,2 & 10,88 & 59,01 & 7,630 & 740,85 & 91,653 & 660,522 \\
\cline { 2 - 8 } & 0,4 & 11,64 & 118,0 & 7,815 & 762,02 & 134,328 & 697,885 \\
\cline { 2 - 8 } & 0,6 & 13,12 & 177,0 & 8,606 & 795,59 & 174,972 & 764,519 \\
\cline { 2 - 8 } & 0,8 & 15,34 & 236,0 & 9,979 & 836,11 & 218,903 & 854,933 \\
\cline { 2 - 8 } & 1,0 & 18,46 & 295,0 & 12,185 & 893,49 & 269,028 & 978,991 \\
\hline
\end{tabular}

В результате проведено 28 расчетов (по 14 на каждую модель самолета). В процессе расчета сходимость решения основных уравнений составила $10^{-4}$. Дисбаланс сходимости уравнений во всех расчетах порядка $0,1 \%$ [8].

На рис. 12 и 13 показано сравнение результатов моделирования лобового сопротивления самолетов с учетом и без учета реактивной тяги двигателей $[19,20]$.
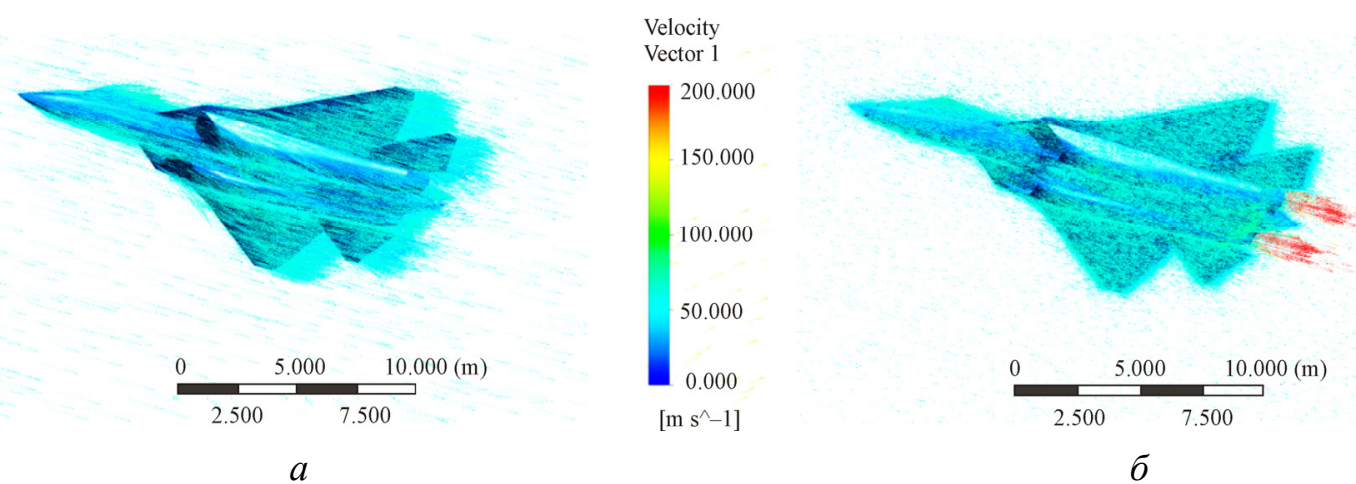

Рис. 12. Векторы скорости для самолета Т-50 с АЛ-41Ф2 при $H=10$ км и $\mathrm{M}=0,2: a$ - моделирование без учета реактивной тяги; $\sigma$ - моделирование с учетом реактивной тяги
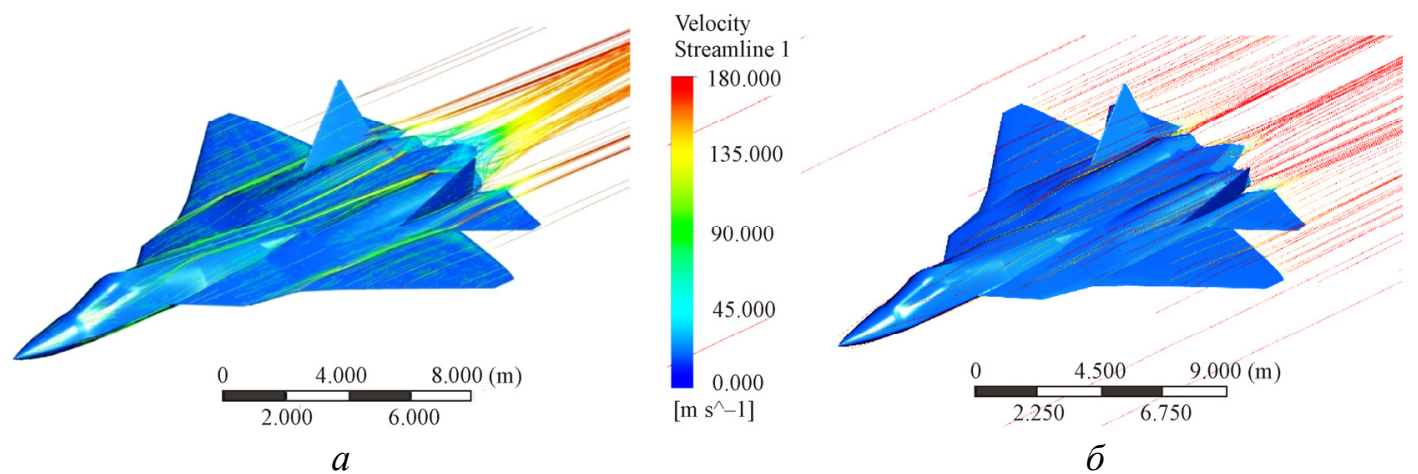

Рис. 13. Линии тока самолета Т-50 с АЛ-41Ф2 при $H=10000$ м и $\mathrm{M}=0,6: a$ - моделирование без учета реактивной тяги; $\sigma$ - моделирование с учетом реактивной тяги 


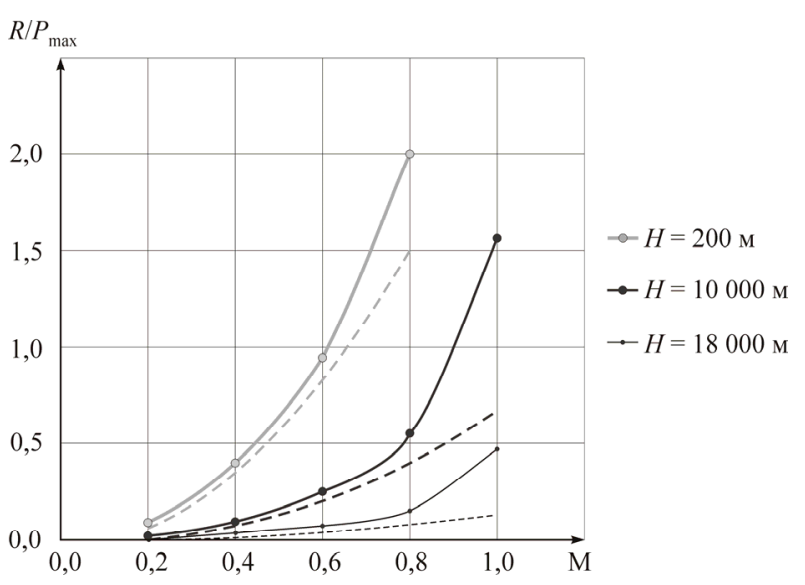

Рис. 14. Зависимость $R / P_{\max }$ от скорости М и высоты полета $H$ при моделировании без и с учетом реактивной тяги двигателей самолета Т-50: — без двигателей; - - с двигателями
На рис. 14 представлено изменение относительного сопротивления моделей самолетов при моделировании без и с учетом реактивной тяги двигателей в зависимости от разных чисел Маха при различных высотах полета.

На графике видно, что относительное сопротивление планеров ЛА при моделировании с учетом реактивной тяги уменьшилось по сравнению с моделированием на первом этапе без учета реактивной тяги (см. рис. 8). При низких скоростях полета (до 0,6 М) результаты расчета изменились в среднем на $12,5 \%$, а при больших скоростях изменение составило от 22 до $70 \%$.

\section{Выводы}

Построены 3D твердотельные модели планеров самолетов Т-50 и F-35B при помощи CAD-системы Autodesk Inventor 2016. Проведено 3D численное моделирование и исследование лобового сопротивления этих моделей без учета работы двигателей на разных высотах и скоростях. Получены значения лобового сопротивления ЛА и найдены их коэффициенты сопротивления.

Разработаны и идентифицированы термогазодинамические модели ТРДДФсм для военных высокоманевренных самолетов V поколения: АЛ-41Ф1, АЛ-41Ф2, F-119 и F-135 в DVIGw на взлетном максимальном и полном форсированных режимах.

Проведен расчет ВСX этих двигателей в диапазоне изменения Маха полета от 0 до 1,2 с шагом 0,2 и изменения высоты полета $H$ от 0 до 20000 м с шагом 2000 м. Получены значения основных параметров рассматриваемых двигателей на различных высотах и скоростях.

Проведено 3D численное моделирование и исследование лобового сопротивлений самолетов Т-50 и F-35B с учетом работы двигателей на разных высотах и скоростях. Получены уточненные значения лобового сопротивления самолетов, найдены погрешности моделирования по сравнению с результатами расчета без учета работы двигателей.

Разработанная методика моделирования обтекания ЛА позволяет заменить модельные исследования в аэродинамической трубе и полнонатурные на летающей лаборатории на 3D численный термогазодинамический расчет, что экономит ресурсы и время исследования.

\section{Библиографический список}

1. Кишалов А.Е., Самигуллин. А.А. Анализ характеристик авиационных ГТД V поколения // Молодеж. вестн. УГАТУ. - Уфа, 2014. - № 2(11). - С. 47-54.

2. Кишалов А.Е., Ключев Н.А. Моделирование и анализ характеристик ТРДДФсм для самолетов V поколения в системе DVIGw // Мавлютовские чтения: материалы X Всерос. молод. науч. конф. / Уфим. гос. авиац. техн. ун-т. - Уфа, 2016. - С. 199-203.

3. Моделирование аэродинамических характеристик современных и перспективных самолетов V поколения при помощи ANSYS CFX / А.Е. Кишалов, Н.А. Ключев, Р.И. Султанов, А.А. Самигуллин // Молодеж. вестник УГАТУ. - Уфа, 2016. - № 2(15). - С. 44-53.

4. Шевелев Н.А., Модорский В.Я. Решение инженерных задач на высокопроизводительном вычислительном комплексе ПНИПУ // Вестник Пермского национального исследовательского политехнического университета. Аэрокосмическая техника. - 2014. - № 4(39). - С. 6-16. 
5. Бутымова Л.Н., Шевелев Н.А., Модорский В.Я. Численное моделирование газодинамических процессов в энергетической установке на базе двигателя Стирлинга // Вестник Пермского национального исследовательского политехнического университета. Аэрокосмическая техника. - 2016. - № 1(44). С. 102-115.

6. Ахмедзянов Д.А., Кишалов А.Е., Маркина К.В. Применение ANSYS CFX в исследовании взаимодействия сверхзвуковой реактивной струи с планером самолета МиГ-21 // Будущее авиации и космонавтики за молодой Россией: материалы междунар. молодеж. форума / Рыб. гос. авиац. техн. ун-т имени П.А. Соловьева. - Рыбинск, 2015. - С. 103-108.

7. Биматов В.И., Фарапонов В.В., Савкина Н.В. Математическое моделирование обтекания тел на основе программного модуля ANSYS CFX // Изв. высш. учеб. завед. Физика. - Томск: Изд-во нац. исслед. Том. гос. ун-та, 2013. - № 6(56). - С. 8-10.

8. Кудоярова В.М., Кишалов А.Е. Решение прикладных задач теплообмена и гидрогазодинамики в пакете ANSYS: учеб. пособие / Уфим. гос. авиац. техн. ун-т. - Уфа, 2016. - 219 с.

9. Клеванский В. М. Гидрогазодинамика: учеб. пособие / Уфим. гос. авиац. техн. ун-т. - Уфа, 2010. - 309 c.

10. Бакулев В.И., Крылов Б.А., Голубев В.А. Теория, расчет и проектирование авиационных двигателей и энергетических установок: учебник. - М: Изд-во МАИ, 2003. - 688 с.

11. Framework CAMCTO for gas turbine engines conceptual design / A.E. Mikhaylov, I.A. Krivosheev, D.A. Akhmedzyanov, A.B. Mikhaylova // The 17th International Workshop on Computer Science and Information Technologies. Italy. - Rome, 2015. - C. 74-78.

12. Власова Е.С., Кишалов А. Е. Параметрическая идентификация математической модели ГТД в системе DVIGwp // Мавлютовские чтения: материалы всерос. науч.-техн. конф. / Уфим. гос. авиац. техн. ун-т. - Уфа, 2007. - С. 56-57.

13. Пугонин К.С. Высотно-скоростные характеристики турбореактивного двигателя // Инновационные процессы в современном мире (Иннофорум-2016): материалы междунар. науч.-практ. конф. / Фонд науки и образования. - Сочи, 2016. - С. 216-217.

14. Разработка методики идентификации математической имитационной сетевой модели ГТД в системе моделирования DVIGw / И.А. Кривошеев, И.М. Горюнов, К.Е. Рожков, Д.А. Кривцов // Современные проблемы науки и образования. - Пенза: Академия естествознания, 2013. - № 4. - 39 с.

15. Ахмедзянов Д.А, Кишалов А.Е. Термогазодинамическое моделирование авиационных двигателей и их элементов: лаб. практикум / Уфим. гос. авиац. техн. ун-т. - Уфа, 2012. - 90 с.

16. Чечулин А.Ю., Кривошеев И.А. Методика учета мощности, затрачиваемой на вращение ротора авиационного двигателя, в нелинейной динамической модели запуска авиационного газотурбинного двигателя // Вестник Пермского национального исследовательского политехнического университета. Аэрокосмическая техника. - 2017. - № 49. - С. 81-94.

17. Ахмедзянов Д.А., Кишалов А.Е. Газодинамическое моделирование в программном комплексе ANSYS CFX и ANSYS Workbench: учеб. пособие / Уфим. гос. авиац. техн. ун-т, - Уфа, 2008. - 45 с.

18. Исследование взаимодействия сверхзвуковой реактивной струи с планером самолета в ANSYS CFX 14.5 / В.С. Жернаков, И.А. Кривошеев, Д.А. Ахмедзянов, А.Е. Кишалов, К.В. Маркина, В.Д. Липатов // Молодеж. вестник УГАТУ. - 2015. - № 1(13). - С. 86-95.

19. Bergeron K., Seidel J., McLaughlin T. Wind Tunnel Investigations of Rigid Ram-Air Parachute Canopy Configurations // $23^{\text {rd }}$ AIAA Aerodynamic Decelerator Systems Technology Conference USA, Daytona Beach, FL, Apr 2015. - C. 13021.

20. Khan O., Masud J. Evaluating an Experimental Streamlined Fairing for a Diverterless Supersonic Inlet (DSI) Equipped Aircraf // 54 ${ }^{\text {th }}$ AIAA Aerospace Sciences Meeting USA, San Diego, California, Jan 2016. C. 13179 .

\section{References}

1. Kishalov A.E., Samigullin A.A. Analiz harakteristik aviacionnyh gtd V pokoleniya [Analysis of characteristics of aviation engine for the fifth generation]. Molodyozhnyj Vestnik Ufimskogo gosudarstvennogo aviatsionnogo tekhnicheskogo universiteta. 2014, no 2(11), pp. 47-54.

2. Kishalov A.E., Klyuchev N.A. Modelirovanie i analiz harakteristik TRDDFsm dlya samolyotov $\mathrm{V}$ pokoleniya $\mathrm{v}$ sisteme DVIGw [Modeling and analysis of the characteristics for aircraft of the fifth generation in the system DVIGw]. Materialy X vserossijskoy molodezhnoy nauchnoy konferencii "Mavlyutovskie chteniya", 2016, pp. 199-203. 
3. Kishalov A.E., Klyuchev N.A., Sultanov R.I., Samigullin A.A. Modelirovanie aerodinamicheskih harakteristik sovremennyhh i perspektivnyh samolyotov V pokoleniya pri pomoshhi ANSYS CFX [Modeling of the aerodynamic characteristics of modern and advanced aircraft fifth generation with ANSYS CFX]. Molodyozhnyj vestnik Ufimskogo gosudarstvennogo aviatsionnogo tekhnicheskogo universiteta. 2016, no 2(15), pp. 44-53.

4. Shevelev N.A., Modorskij V.Y. Reshenie inzhenernyh zadach na vysokoproizvodi-telnom vychislitel'nom komplekse PNIPU [Solution of engineering tasks on vysokoproizvoditel-tional computing complex of PNRPU]. Bulletin of Perm National Research Polytechnic University. Aerospace Engeneering, Perm, PNRPU, 2014, no 4(39), pp. 6-16.

5. Butymova L.N., Shevelev N.A., Modorskij V.Y. Chislennoe modelirovanie gazodi-namicheskih processov v ehnergeticheskoj ustanovke na baze dvigatelya Stirlinga [Numerical simulation of gas dynamic processes in power plant based on Stirling engine] Bulletin of Perm National Research Polytechnic University. Aerospace Engeneering, Perm, PNRPU, 2016, no 1(44), pp. 102-115.

6. Akhmedzyanov D.A., Kishalov A.E., Markina K.V. Primenenie ANSYS CFX v issledovanii vzaimodejstviya sverhzvukovoj reaktivnoj strui s planerom samoleta MiG-21 [The use of ANSYS CFX to study the interaction of a supersonic reagent-tion of the jet with the airframe of the MiG-21]. Materialy mezhdunarodnogo molodezhnogo foruma "Budushhee aviacii i kosmonavtiki za molodoj rossiej". Rybinsk, RSATU named after P.A. Solovyova, 2015, pp. 103-108.

7. Bimatov V. I., Faraponov V. V., Savkina N. V. Matematicheskoe modelirovanie obtekaniya tel na osnove programmnogo modulya ansys cfx [Mathematical modeling of flow around bodies on the basis of the software module ANSYS CFX]. Izvestiya vysshix uchebnyx zavedenij. fizika. nauchnyj zhurnal. Tomsk, NRTSU, 2013, no 6(56), pp. 8-10

8. Kudoyarova V.M., Kishalov A.E. Reshenie prikladnyh zadach teploobmena i gidrogazodinamiki v pakete ansys [The decision of applied problems of heat transfer and fluid dynamics in ANSYS]. Ufa, USATU, 2016, 219 p.

9. Klevanskij V.M. Gidrogazodinamika [Fluid dynamics]. Ufa, USATU, 2010, 309 p.

10. Bakulev, V.I., Krylov B.A., Golubev V.A. Teoriya, raschyot i proektirovanie aviacionnyh dvigatelej i energeticheskih ustanovok [Theory, calculation and design of aviation engines and power installations]. Moscow, MAU, 2003, 688 p.

11. Mikhaylov A.E., Krivosheev I.A., Akhmedzyanov D.A., Mikhaylova A.B. Framework camcto for gas turbine engines conceptual design. The 17 th international workshop on computer science and information technologies, Rome, Italy, 2015, pp. 74-78.

12. Vlasova E.S., Kishalov A.E. Parametricheskaya identifikaciya matematicheskoj modeli gtd v sisteme DVIGwp [Parametric identification of a mathematical model of GTE in the system DVIGwp]. Vserossijskaya NTK “mavlyutovskie chteniya”. Ufa, USATU, 2007, pp. 56-57.

13. Pugonin K.S. Vysotno-skorostnye harakteristiki turboreaktivnogo dvigatelya [Altitude-speed characteristics of turbojet engine]. Materialy mezhdunarodnoj nauchno-prakticheskoj konferencii innoforum-2016. Sochi, 2016, pp. 216-217

14. Krivosheev I.A., Goryunov I.M., Rozhkov K.E., Krivcov D.A. Razrabotka metodiki identifikacii matematicheskoj imitacionnoj setevoj modeli gtd v sisteme modelirovaniya DVIGw [The development of methods of identification of mathematical simulation of the network GTD model in the system simulation DVIGw]. Sovremennye problemy nauki i obrazovaniya. nauchnyj zhurnal. Penza, akademiya estestvoznaniya, 2013, no 4, 39 p.

15. Akhmedzyanov D.A, Kishalov A.E. Termogazodinamicheskoe modelirovanie aviacionnyh dvigatelej $\mathrm{i}$ ix elementov [Thermodynamic modeling of aircraft engines and their components]. Ufa, USATU, $2012,90 \mathrm{p}$.

16. Chechulin A.Y., Krivosheev I.A. Metodika ucheta moshchnosti, zatrachivaemoj na vra-shchenie rotora aviacionnogo dvigatelya, v nelinejnoj dinamicheskoj modeli zapuska aviacionnogo gazoturbinnogo dvigatelya [Methodology of power expended on the rotation of the rotor of aircraft engine, in a nonlinear dynamic model introduces the ka aircraft gas turbine engine]. Bulletin of Perm National Research Polytechnic University. Aerospace Engeneering, Perm, PNRPU, 2017, no 49, pp. 81-94.

17. Akhmedzyanov D.A., Kishalov A.E. Gazodinamicheskoe modelirovanie v programmnom komplekse ANSYS CFX i ANSYS workbench [Gas dynamic simulation in ANSYS CFX and ANSYS Workbench]. Ufa, USATU, 2008, 45 p.

18. Zhernakov V.S., Krivosheev I.A., Akhmedzyanov D.A., Kishalov A.E., Markina K.V., Lipatov V.D. Issledovanie vzaimodejstviya sverhzvukovoj reaktivnoj strui s planerom samolyota v ANSYS CFX 14.5 [Study 
of the interaction of supersonic jet with the airframe itself-summer in ANSYS CFX 14.5]. Molodyozhnyj vestnik ugatu. ezhemesyachnyj nauchnyj zhurnal. Ufa, USATU, 2015, no 1(13), pp. 86-95.

19. Bergeron K., Seidel J., McLaughlin T. Wind Tunnel Investigations of Rigid Ram-Air Parachute Canopy Configurations. $23^{\text {rd }}$ AIAA Aerodynamic Decelerator Systems Technology Conference USA, Daytona Beach, FL, Apr 2015, p. 13021.

20. Khan O., Masud J. Evaluating an Experimental Streamlined Fairing for a Diverterless Supersonic Inlet (DSI) Equipped Aircraf. 54 ${ }^{\text {th }}$ AIAA Aerospace Sciences Meeting USA, San Diego, California, Jan 2016, p. 13179.

\section{Об авторах}

Кишалов Александр Евгеньевич (Уфа, Россия) - кандидат технических наук, доцент кафедры «Авиационная теплотехника и теплоэнергетика» Уфимского государственного авиационного технического университета (450008, г. Уфа, ул. Карла Маркса, д. 12, e-mail: kishalov@ufanet.ru).

Ключев Никита Александрович (Уфа, Россия) - магистрант кафедры «Авиационная теплотехника и теплоэнергетика» Уфимского государственного авиационного технического университета $(450008$, г. Уфа, ул. Карла Маркса, д. 12, e-mail: kluchev-nikita@ yandex.ru).

\section{About the authors}

Aleksandr E. Kishalov (Ufa, Russian Federation) - Ph. D. in Technical Sciences, Associate Professor, Department of Aviation of Thermal Engineering and Heat Power Engineering Ufa State Aviation Technical University (USATU) (12, K. Marx st., Ufa, 450008, Russian Federation, e-mail: kishalov@ufanet.ru).

Nikita A. Klyuchev (Ufa, Russian Federation) - Master Stydent, Department of Aviation of Thermal Engineering and Heat Power Engineering Ufa State Aviation Technical University (USATU) (12, K. Marx st., Ufa, 450008, Russian Federation, e-mail: kluchev-nikita@yandex.ru).

Получено 14.09.2017 